\title{
The bits and bytes of computer/Internet addiction: A factor analytic approach
}

\author{
MARC E. PRATARELUI, BLAINE L. BROWNE, and KIMBERLY JOHNSON \\ Oklahoma State University, Stillwater, Oklahoma
}

\begin{abstract}
A factor analytic approach was used to explore possible constructs underlying so-called computer/Internet addiction. A 94-item survey was developed, from which two major and two minor factors were derived. Factor 1 focused on problematic computer-related behaviors in heavy users of the Internet, whereas Factor 2 focused on the usefulness and general purpose nature of computers and the Internet. Factor 3 isolated a combination of use of the Internet for sexual gratification and shyness/introversion, and Factor 4 focused on an absence of problems related to Internet use that were coupled with a mild aversion or a disinterest in this technology. These data support the notion that some individuals have a mixture of obsessive-like characteristics related specifically to their computer/Internet use but that, not surprisingly, they also exhibit a preference for on-line, rather than in-person, interactions.
\end{abstract}

The 1990s have been distinguished by the explosive growth of the Internet, surpassed in magnitude and scope only by the development, affordability, and availability of the personal computer. In the absence of hourly charges and given the ease of unlimited access to the Internet, individuals have been able to $\log$ on for hours at a time. However, like any technology aimed specifically for human consumption, abuse can have severe consequences for the individual and, potentially, for the community at large (Anderson, Bikson, Law, \& Mitchell, 1995; Jacobson \& Roucek, 1959; King \& Kraemer, 1995; Kraut et al., 1998; Young, 1996). The potential misuse (i.e., excessive use) of computers and, in particular, of the Internet has led to concern over the addictive properties of this new technology (see Fizel, 1997; Griffiths, 1995a, 1995b, 1997, 1998; Shaffer, as cited in O'Neill, 1995). A new jargon has allowed frequent users to sometimes refer to themselves as webaholics or cyberholics. Arguably, within a few decades of the introduction and proliferation of television, social critics, educators, clinicians, and politicians began the ongoing debate as to its contribution to the decline of the family and morality and to other more tangible social ills. Similarly, some have reported that the computer and, more

\footnotetext{
We thank John Chandler and his wife and son for their help in designing and revising the survey instrument, along with the students and computer laboratory assistants in the Math/Sciences building at Oklahoma State University. We also thank Pat Murphy for sharing his thoughts during the development of the study. We appreciate the help of Dan Mason and Amy Tiedt for the many hours of data entry and editing of the manuscript and the reviews and comments of Nancy Duncan, Mark Griffiths, two anonymous SCiP and BRMIC reviewers, as well as those of the editor on earlier versions of the manuscript. We also wish to recognize several of the authors of Web pages, from which ideas for some of the survey items originated. Correspondence or requests for the survey materials should be addressed to M. E. Pratarelli, 215 North Murray, Department of Psychology, Oklahoma State University, Stillwater, OK 74078 (e-mail: marcp@okway.okstate.edu).
}

recently, the Internet and the World-Wide Web (WWW) are also altering the social landscape (see, Orzack, 1998; Shotton, 1989; Sleek, 1998).

A fairly recent development in the debate is the appearance of a cluster of behaviors potentially causing problems, variously labeled computer or Internet addiction or dependency, Internet addictive disorder, or cyberaddiction (Brenner, 1997; Fearing, 1997; Henderson, 1996; Orzack, 1998; Shotton, 1989, 1991; Young, 1996). One important issue that has not been sufficiently well addressed is the classic interdependency between the medium and the message. Early research (e.g., Shotton, 1989, 1991) - prior to the popularization of the Internet, when computer use required a more sophisticated user (e.g., one with knowledge of DOS or UNIX commands) - clearly showed that much of the fascination or obsession had to do with the medium. On the basis of more recent research, if one assumes that the computer remains the medium, and the Internet a convenient metaphor for the sum of the messages, then one could argue that the fascination or obsession has more to do with the message. However, because the recent research either uses the terms interchangeably or uses one to the exclusion of the other, without differentiating as to whether the symtomology is specifically attributable to the medium or the message, a more conservative approach would be to use the terms together. This should continue until such time that specific attributes of the putative disorder can be assigned either to the medium or to the message. Thus, for the purpose of focusing our present understanding of the behavior we sought to investigate, we operationally defined computer/Internet addiction as a putative phenomenon or disorder that is dependent on both the medium and the message, without exhibiting a bias in favor of either.

Although some suggest that the addiction label is premature (Brenner, 1997), many have individually verified its existence through subjective surveys or personal obser- 
vation. In some cases, addiction tests, support services, or clinical treatments have been developed (see, e.g., Fearing, 1997; Orzack, 1998; Young, 1996). Nonetheless, treating an ill-defined, potentially misclassified dysfunctional behavioral profile has become a lucrative business on the Internet. Although some researchers have attempted to validate computer/Internet addiction by surveying computer users (Brenner, 1997; Young, 1996), no attempts have been made to characterize empirically the psychological factors that may drive the problem behavior in certain individuals. Such validation is required before determining whether a problem behavior for an individual constitutes a unique disorder. Moreover, little more than anecdotal evidence and intuitive rationalizations based on descriptive statistics has been offered when discussions turn to the prediction of which individuals are most at risk of developing an addiction (Fearing, 1997; Roberts, 1997; Young, 1996). In most cases, studies dealing with computer/Internet addiction are not empirical studies but, rather, newspaper op-eds, periodical articles, or subjective commentary on personal WWW sites, with personal opinions being based on introspection or intuitive rationalizations; one Web site actually cites the advice of a prominent newspaper columnist of the "Dear Abby" type.

Young (1996) and Griffiths (1998) used a different approach by examining and discussing the value of case studies of individuals purportedly addicted to computer or Internet use. As with all case studies, although the data are often provocative and sometimes insightful, they are less than satisfactory in terms of providing generalizable models. Nonetheless, Griffiths (1998) most recently has summarized the preponderance of the evidence as suggesting "that excessive usage in a majority of cases appears to be purely symptomatic but that for what appear to be an exceedingly tiny minority, the Internet may be addictive" (p. 71). Therefore, in view of the growing concern over the addictive properties of computers and the Internet and in view of the accumulating anecdotal evidence and intuitive rationalizations, the major goal of the present study was to begin filling the gap created by the absence of empirical evidence supporting or disconfirming a cluster of behaviors unique to these combined technologies. The specific objectives were to examine and test the efficacy of several factors purported to drive the apparent craving or compulsion in computer/Internet use and to explore some of the possible personality attributes that may predispose some individuals to this particular type of behavior profile.

\section{METHOD}

\section{Subjects}

The principal focus of our concern was the traditional college student population. Three hundred and fifty subjects were recruited from various departments at Oklahoma State University. From these subjects, 341 surveys (163 from males, 178 from females) were completed and found to be free of artifacts (e.g., malingering, incomplete sections, etc.). The subjects were offered extra course credit for their participation in filling out the anonymous survey. Mean age was 22.8 years $(S D=5.88$ years), with a range from 16 to 67 years; the median age was 21 years. The purpose of sampling from across several majors was to enhance the likelihood of recruiting individuals with specific interests involving computers while approximating a more diverse and representative sample. Moreover, not recruiting from at least one area in which computer enthusiasts congregate in larger than normal numbers would have underrepresented their numbers in the sample. In all, 103 surveys were collected from students attending math and computer science courses, and 238 were collected from the sociology and psychology departments. The sampling manipulations are not intended to represent the normal population, because the sample came from the university environment.

\section{Materials and Procedures}

A survey was developed, focusing on conventional demographic (categorical) variables and on domain-specific variables regarding the computer, the Internet, and their use. The survey consisted of 19 such categorical items and 74 dichotomous (true/false) items addressing a broad range of computer/Internet, social, and personal activities. Three of the true/false items were used as foils (e.g., "I have never met anyone who wears glasses or contacts") to detect malingerers in the sample. The working logic of using so many variables was to develop an initial survey that was comprehensive and from which insensitive variables could later be discarded, but only on an empirical basis. The Appendix lists the various categorical and dichotomous variables of the survey that were used; it is the original survey, as it was administered to the sample in the present database. In addition to the present authors, two professors from computer science, three self-diagnosed computer addicts, two computer laboratory supervisors who commented on the nighttime behavior of subjects, and a university counseling psychologist who reported having treated several computer/Internet-addicted students assisted in the development of the survey instrument. The survey was also based on a meta-analysis of the existing literature and of surveys on computer or Internet use and addiction (including tests and surveys that were found on the Internet). Many features found consistently were included as specific questions in the present survey. Because there were no valid and reliable empirically tested surveys, face validity was the sole criterion for including a variable during the development of the instrument. In nearly all cases, instructions for completing the anonymous survey were given to groups; $15 \mathrm{~min}$ were allotted, with specific instructions not to talk and not to return to previous items once a response had been entered.

\section{RESULTS}

The survey responses were screened for missing data and for normality. Frequencies were computed for the functional demographic variables. All the variables were transformed to $z$-scores, for ease of comparison. A principal components factor analysis was performed to explore the interrelationships between the categorical and the dichotomous variables. A total of $31 \%$ of the variance was explained with four factors. Fifty-five of the 93 initial variables were isolated on the basis of sufficient variability. Varimax rotation was used for ease of interpretation of the factors. A minimum criterion of .35 was set as the cutoff at which to accept individual factor loadings. Table 1 lists the factor loadings for the two principal factors ( 1 and 2) and two additional minor factors ( 3 and 4 ).

Factor 1 accounted for $17.9 \%$ of the variance, with an eigenvalue of 11.26. Twenty-three of the true/false items 
Table 1

Individual Factor Loadings for the Two Main Factors (1 and 2$)$ and the Two Minor Factors ( 3 and 4$)$

\begin{tabular}{|c|c|c|c|c|}
\hline \multirow[b]{2}{*}{ Item } & \multicolumn{4}{|c|}{ Factor } \\
\hline & 1 & 2 & 3 & 4 \\
\hline Played video games (2) & & .50269 & & \\
\hline Late for meetings (3) & .49963 & & & \\
\hline Prefer being on line (5) & .61398 & & & \\
\hline Lost sleep (6) & .49845 & & & \\
\hline Missed meals (7) & .54305 & & & \\
\hline Meet Net friends (8) & & & & -.39585 \\
\hline Alleviate depression (9) & .43828 & & & \\
\hline Access was down (11) & & .45624 & & \\
\hline Alleviate loneliness (12) & .40095 & & & \\
\hline Lose track of time $(20)$ & .52657 & & & \\
\hline People say I'm shy (21) & & & .56231 & \\
\hline Don't miss the Net (23) & & & & .46368 \\
\hline On line too long (24) & .57435 & & & \\
\hline Called "nerd," etc. (25) & .53490 & & & \\
\hline Use adult only sites (26) & & .40617 & .55004 & \\
\hline Introverted (28) & & & .58928 & \\
\hline Dietary changes (38) & .68564 & & & \\
\hline Internet use is okay (39) & & & & .36656 \\
\hline Can't stay off line (41) & .58430 & & & \\
\hline I exercise less now (42) & .63639 & & & \\
\hline Tried new things (43) & .63019 & & & \\
\hline Physically aroused (44) & .35581 & & .43743 & \\
\hline Found info on line (49) & & .39185 & & \\
\hline Borrowed money (50) & & & & .55711 \\
\hline Extroverted (54) & & & -.55780 & \\
\hline Cut short my sleep (55) & .66620 & & & \\
\hline Government regulation (58) & & & .49679 & \\
\hline Prefer face-to-face (59) & & & -.38186 & .37627 \\
\hline Work is okay $(60)$ & & & & .43847 \\
\hline No Internet friends (61) & & & & .47099 \\
\hline No access is okay (62) & & & & .35460 \\
\hline Downloaded nudes (64) & & .35871 & .56837 & \\
\hline Gambling (65) & .43214 & & & \\
\hline Stay connected (66) & .47995 & & & \\
\hline Computer programs (67) & .35771 & & & \\
\hline Hacked my way in (68) & .50906 & & & \\
\hline Disorientation (69) & .50412 & & & \\
\hline Finding new sites $(70)$ & .45609 & & & \\
\hline Shopping on line (71) & & .42681 & & \\
\hline Finding answers ( 72$)$ & & .38690 & & \\
\hline Used encryption (73) & .41999 & & & \\
\hline Use e-mail extensively & & .52040 & & -.35445 \\
\hline Use FTP extensively & & .51274 & & \\
\hline Many hours/week on line & .43125 & .67487 & & \\
\hline IRC (monitor chat lines) & & .42479 & & \\
\hline Like Playing MUD & & .40000 & & \\
\hline Access during the day & & .51213 & & \\
\hline Log on from home & & -.47456 & & \\
\hline Access from campus & & .44831 & & \\
\hline Extensive WWW use & & .70324 & & \\
\hline
\end{tabular}

Note-The item description and the number (in parentheses) listed on the left correspond to the item and number in the 74 true/false items in the survey. The nine categorical measures listed at the bottom of the table correspond to the matching variable in the first section of the survey. The criterion for acceptance was .35 or higher. Positive loadings represent a positive relationship for the corresponding variable, and a negative loading suggests that the variable is orthogonally opposed to the factor.

loaded onto the first factor, along with one categorical variable that was negatively loaded. The second factor accounted for $6 \%$ of the variance, with an eigenvalue of 3.79 . Seven true/false items loaded onto this factor, along with one positively and eight negatively loaded categorical variables. The third and fourth factors accounted for only $3.8 \%$ and $3.4 \%$ of the variance, respectively, but they were included because they appeared to be related to the main construct (problems with computers/Internet) when the specific variables were examined. For Factor 3, six true/ false items were positively loaded, and two were negatively loaded; no categorical variables loaded onto Factor 3. Factor 4 had eight positively and two negatively loaded true/false variables and no categorical variables.

\section{DISCUSSION}

\section{Factor 1}

The principal functional characteristic of these variables suggests a construct that is aimed at problem behavior and the hard-core computer/Internet user. Referencing the listing in Table 1 with the corresponding items in the survey revealed the complex and diverse nature of this major factor. The frequency of responses for individual elements (Table 2) ranged from $4 \%$ to $24 \%$ of the sample, with factor loadings ranging from approximately .4 to .7. In general, the first factor is characterized by the subjects' reports of loneliness and social isolation, of forgetting to eat or being late and missing appointments because of their heavy use of the Internet, of being called "junkie" or "computer nerd," of negative changes in dietary and exercise habits, and of eye strain. Of particular concern is the report that, in view of the loneliness and social isolation owing to their computer/Internet use, approximately $23 \%$ of the sample reported using the Internet "to feel better" and to alleviate feelings of depression. Congruent with this effect are responses to the item that indicate a preference for being on line rather than socializing with people in vivo. The reason a depressed individual seeks out the Internet to alleviate feelings of depression is not clear from the present database. Kraut et al. (1998), however, have made the causal claim that it is Internet use that leads to feelings of isolation and depression. They noted that "The major threat to the causal claim would arise if some unmeasured factor varying over time within individuals were to simultaneously cause increases in their use of the Internet and declines in their normal levels of social involvement and psychological well-being" (p. 1028). The present database clearly shows that, in view of Factor 1, which focuses on negative aspects of computer/Internet use, some individuals will increase their activity as their feelings of isolation increase. This form of compensatory behavior produces a cycle in which loneliness and depression feeds computer/Internet use, which, in turn, leads to more loneliness and depression, which, in turn, feeds the computer/Internet use, and so forth. Anecdotal comments from counselors and selfidentified addicted individuals are consistent with this interpretation of the present data.

Nearly $20 \%$ of the sample reported using the Internet to become sexually aroused. A small proportion (4\%) reported using the Internet for gambling purposes. Yet another characteristic of this principal factor was that ap- 
Table 2

\section{Percentage of 341 Subjects Who Responded} to Each of the True/False Statements That Had Positive Loadings for Factors 1-4

\begin{tabular}{|c|c|c|c|c|c|c|c|}
\hline \multicolumn{8}{|c|}{ Factor } \\
\hline \multicolumn{2}{|c|}{1} & \multicolumn{2}{|c|}{2} & \multicolumn{2}{|c|}{3} & \multicolumn{2}{|c|}{4} \\
\hline Item & $\%$ & Item & $\%$ & Item & $\%$ & Item & $\%$ \\
\hline 3 & 13 & 2 & 58 & 21 & 30 & 23 & 75 \\
\hline 5 & 6 & 11 & 66 & 26 & 34 & 39 & 48 \\
\hline 6 & 20 & 26 & 34 & 28 & 32 & 51 & 51 \\
\hline 7 & 21 & 49 & 62 & 44 & 20 & 59 & 77 \\
\hline 9 & 23 & 64 & 36 & 58 & 24 & 60 & 81 \\
\hline 12 & 18 & 71 & 20 & 64 & 36 & 61 & 61 \\
\hline 20 & 11 & 72 & 40 & & & 62 & 70 \\
\hline
\end{tabular}

$\begin{array}{ll}24 & 14 \\ 25 & 15\end{array}$

$\begin{array}{rr}25 & 15 \\ 48 & 9\end{array}$

416

$42 \quad 8$

$43 \quad 11$

$44 \quad 20$

$55 \quad 8$

$58 \quad 24$

$65 \quad 4$

$66 \quad 11$

$67 \quad 14$

$68 \quad 13$

6923

$70 \quad 24$

$73 \quad 12$

Note-The item numbers to the left of the percentages correspond to the labels listed in Table 1.

proximately $13 \%$ of the subjects classified themselves more or less as hackers; several other variables that loaded onto this factor are consistent with this self-report-that is, they were characteristic of the sophisticated user capable of writing software programs beyond what is required for class assignments or work and of sending encrypted messages and who engages in frequent use of e-mail and file transfer protocol and in more-than-average use of multiple-user domain (MUD) games. Another notable feature of this principal factor was that $28 \%$ of the sample reported concern over government involvement in regulating the Internet. Similarly, interviews with students who self-identified as being frequent Internet users, or individuals for whom Internet use was excessive and caused problems, revealed their irritation with the university's restrictions on their access to Internet sites containing sexually graphic material. Interestingly, the same individuals commented that this action merely forced them to access such sites from home or work.

Finally, the number of hours per week of computer/Internet use was negatively loaded onto Factor 1, indicating that the subjects responding with this pattern tended to respond on the high end of this scale. The upper $26 \%$ of the sample reported usage above $10-20 \mathrm{~h}$ per week, with $3 \%$ claiming $40 \mathrm{~h}$ or more per week. It is intuitively obvious that, to have as many experiences as are covered by the Factor 1 loadings, the subjects would have to be spending a large amount of time on line. Thus, another aspect of problem computer/Internet use is the sheer amount of time spent. As others have noted, spending time engaged on the computer takes away opportunities to socialize that would otherwise have been available (Anderson et al., 1995; Healy, 1998; Kraut et al., 1998; Sproull \& Faraj, 1995). Kraut et al. have shown recently that, as Internet use increased in their longitudinal study sample, happiness and social support decreased, and depression and loneliness increased. Alternatively, others have argued that the Internet actually fosters social interactions (Katz \& Aspden, 1997; Rheingold, 1993). Table 2 lists the percentages of subjects who responded to the specific items loaded onto Factor 1.

\section{Factor 2}

Seven items loaded significantly onto Factor 2 , whose focus was the utilization and usefulness of computer technology in general and of the Internet in particular. The high percentages of responses on these seven items suggest that the functional construct for this factor is the value the subjects placed on the computer and the Internet's general purpose/functional nature. There is a conspicuous absence of problems related to computer/Internet use with this factor. The percentages in Table 2 range from $20 \%$ to $66 \%$ of the subjects responding to these items. Although $34 \%-36 \%$ reported using the Internet to view sexually explicit material, the item that emphasized sexual arousal did not load onto this factor, further indication that this factor does not concern itself with the excesses of computer/Internet use. As Table 2 illustrates, the subjects reported using the Internet for ordering merchandise or asking questions they could have had answered by going to the library; particularly notable, $62 \%$ responded yes to the statement "I have found information or software that I needed free on the net."

\section{Factor 3}

The third and fourth factors explained comparatively little of the variance, but they represent a large percentage of responses, and they focus on two additional unique constructs. Nonetheless, the third factor is characterized by two principal aspects, one functional and the other trait based. Three of the six items that loaded onto Factor 3 are concerned with the use of the Internet for sexual gratification. Two of the remaining three items are concerned with being shy or introverted. ${ }^{1}$ The last item involves concern over government attempts at regulating the Internet. This factor is particularly notable in light of the public, institutional, and governmental reactions over the availability, access, and viewing of sexually explicit material. Between $20 \%$ and $36 \%$ of the 341 respondents had these particular attributes and opinions regarding access and use of sexual material. However, it is interesting to note that only one of these items, that dealing with having become 
sexually aroused while on line, loaded onto Factor 1 , which was concerned with the negative aspects of computer/ Internet use.

That the sexual applications of computer/Internet use load separately onto their own minor factor, whereas only a single sex-related item appears on Factor 1, suggests that the concern or publicity over this issue is highly inflated and probably unjustified for the college-age population; it explains only $3 \%$ of the variance in this sample. These statistics show that, although approximately one third of this college-age group reports having used the Internet to view material of a sexual nature, a considerably smaller proportion report having used it to become aroused. Recall that, in Factor 1, although $20 \%$ reported having been aroused while on line, a smaller fraction of the sample (e.g., between $4 \%$ and $20 \%$ ) reported that their computer/Internet use caused problems to the point of affecting their dietary, exercise, health, sleeping, and social habits. Moreover, personal communications with counseling psychologists suggest that the number of students who are sought out because of their poor scholastic performance or who seek out help themselves because of their excesses of computer/Internet use is currently extremely small (i.e., less than $1 / 2$ of $1 \%$ of the student population in any given year). If this problem behavior arises disproportionately in the high-frequency users who also happen to be shy and introverted, some concern should be focused on examining the coming generation of college students. In this group, currently in the $\mathrm{K}-12$ system, the number of households with computer/ Internet access is currently reported to be as high as 70 million (Sleek, 1998), although clearly only a fraction of these households actually have children in $\mathrm{K}-12$. If one takes an extremely conservative estimate of only 10 million households at present, some study is warranted. In any case, our present data suggest that the alarmist concerns (e.g., Healy, 1998), although warranting future investigation, are probably overrated. Historically, such reactions are typical when a new technology presents itself for mass consumption by the public.

The concern over personality traits that may predict who is predisposed to becoming computer/Internet addicted tends to focus on the shy, introverted, or socially withdrawn individuals (see Douse \& McManus, 1993; Fearing, 1997; Orzack, 1998; Shotton, 1989; Young, 1996). Douse and McManus specifically focused on players of fantasy role-playing games (FRPGs) and compared them on certain personality measures and demographic variables. Their study found that the majority of players were "male, of high educational level, and sharing common interests in FRPGs ... and in computer games in general" (p. 507). On the Bem Sex Role Inventory, players' responses were equivalent on masculinity but lower on the femininity and androgynous subscales, as compared with controls. Players also had lower scores on a measure of empathetic concern. On the basis of their parametric analysis, they referred to the player of FRPGs as "the shy, introverted intellectual." A cursory survey we conducted early in our study of the many WWW pages advertising treatment or diagnosis of computer/Internet addiction revealed a preponderance of anecdotal evidence focused on shy, introverted, and socially withdrawn individuals. Nonetheless, Factor 3 of the present analysis, although a lesser component, suggests that sexual gratification, together with perceived shyness and a preference for personal engagement rather than interpersonal interactions, constitutes one focus of a construct describing some users.

\section{Factor 4}

Ironically, this Factor 4 isolates a construct in which there is an absence of concern over problem use. Items loading onto this factor include (1) the notion that the Internet is impersonal, (2) a preference for face-to-face rather than computer/Internet interactions, and (3) no desire to meet "net friends" in person. Other indicators include the lack of concern over an inability to connect to the Internet and not having had any problems with computer/Internet use $(60 \%)$. Although this factor explains only a small portion of the variance, it is important to remember that the design of this survey, like so many others, is focused on problem behaviors and concerns. Had the design of the statements and questions been focused on exploring positive attributes of computer/Internet use, the major factor (given that an effect exists) would follow the variance generated by the majority of the statements. At present, our focus was on the negative attributes of computer/Internet use, but a reasonable balance needed to be achieved between positive and negative attributes of computer/Internet use.

\section{CONCLUSIONS AND FUTURE CONSIDERATIONS}

The results of the factor analytic approach taken in the present study permits certain conclusions to be summarized from an empirical position. The first factor clearly illustrates that there indeed exists a loosely connected cluster of behaviors that are perceived by many users and others as causing problems in their daily lives. Factor 2 revealed the usefulness of computers and the Internet, whereas Factor 3 was concerned with sexual gratification and shyness as a self-reported trait in some users. The fourth factor revealed a component of computer/Internet use that also focused on negative characteristics, but from respondents for whom computers and the Internet did not pose a problem. Moreover, Factor 4 reflects a mild aversion to using computers and the Internet. (Since computerphobia exists in some small proportion even among college students, we can assume that some of this variance may be attributable to this effect.) Factors 1 and 4 demand more thorough investigation, in view of one of the most compelling characteristics of the Internetnamely, that it was created and grows because it is a plat- 
form for social interaction (Rheingold, 1993). The degree of engagement, of course, is left to the discretion of the consumer.

A problem arises, however, in a select few individuals for whom the technology's implicit ability to become a substitute for human interaction helps them create their own virtual world and virtual reality. In view of recent findings (Kraut et al., 1998) that almost any type of use of the Internet, social or otherwise, may be associated with increases in social isolation and depression for most people, the extreme effects that purportedly lead to addiction should be considered carefully. This should begin with a careful and thorough analysis of what constitutes an $\mathrm{ad}$ diction, as Griffiths (1998) has attempted to do. The first question that inevitably arises is whether computers or the Internet can be addicting, because addictions have traditionally been reserved for biologically verifiable conditions that lead to dependency - for example, nicotine and smoking, cocaine or alcohol and drug abuse. With small variations on the same theme, dictionaries define addiction, addicted, or addict as habitual behavior usually related to a drug. However, synonyms for the term addicted include accustomed, habituated, inclined, prone, and attached. Clearly, any of these synonyms may better describe what excessive use of the computer and the Internet represents: an obsession with a particular nonorganic object or phenomenon, akin to pathological gambling or compulsively making purchases on the home shopping network.

Griffiths (1996) has noted that "technological addictions are operationally defined as non-chemical (behavioural) addictions which involve human-machine interaction" (p. 32). The Diagnostic and Statistical Manual of Mental Disorders-4th edition (DSM-IV) does not recognize computer/Internet or technological addictions. Whether computer/Internet addiction will be included in some future editions as a legitimate diagnosis is open to speculation and will ultimately depend on the consistent observation of a cluster of behaviors that can be operationally defined and reliably measured. If computer/ Internet addiction cannot be isolated as a unique behavioral profile and diagnosis apart from the technology that is the focus of the obsession, compulsion, or dependency, it will likely exist merely as a unique or secondary manifestation of obsessive-compulsive disorder.

Currently, there is considerable evidence to suggest that the degree and manner in which an individual uses computers and the Internet are attributable to little more than personal preference and choice. Whether some of these individuals are addicted is difficult to say, because the usefulness and applicability of the term addiction to computers and the Internet is still unclear and the pattern of usage and of its negative impact on life is mixed, suggesting that not all users have problems with the technology. What is needed is more empirical validation of the behavioral profiles of obsessive and compulsive behaviors related to computers and the Internet, as well as more de- tailed analysis of at-risk populations and individual differences. A much better understanding of the perceptual characteristics and the dynamic functional properties of Internet sites that compel some individuals to remain logged on for hours at the expense of other activities is also needed.

Our current efforts are focused on more discrete post hoc data analyses and on continued refinement of the survey instrument, to enhance our understanding of computer/Internet-related consumerism in subpopulations of college students, as well as in children. One modification that is currently being examined is the use of a Likert-style rating score, as compared with the forcedchoice true/false responses reported in this study. With regard to children, however, another database being developed in our ongoing study in this area is focused on the coming generation of college students. We hypothesize that, as the number of students entering college who use the Internet as part of a daily regimen increases, and assuming that the Internet continues to diversify by exploring new opportunities for social interaction, the number of variables for which obsessive and compulsive behaviors can form will increase. Given the biological and developmental constraints of the human brain, it would be prudent to ask what might be the effects on young children exposed to this rich technology from the moment they have the necessary motor skills to press a button, manipulate a mouse, touch a screen, or speak into a microphone. In educational circles, it is already drawing some concern (Healy, 1998), but it is a concern that should be weighed with regard to the most prudent and efficacious use of the technology.

\section{REFERENCES}

Anderson, R. H., Bikson, T. K., LAW, S. A., \& Mitchell, B. M. (Eds.) (1995). Universal access to e-mail: Feasibility and societal implications. Santa Monica, CA: Rand Corporation.

BRENNER, V. (1997). An initial report on the on-line assessment of internet addiction: The first 30 days of the Internet usage survey [Online]. Available: http://www. ccsnet.com/prep/pap/pap8b/638b012.txt

Douse, N. A., \& McManus, I. C. (1993). The personality of fantasy. British Journal of Psychology, 84, 505-509.

FEARING, J. (1997). Hooked on the Net [On-line]. Available: http:// www.nationalcounseling.com/cmpadict.html

Fizel, D. (1997). Pathological internet use: Psychologists examine who is hooked on the Net and why [On-line American Psychological Association news release]. Available: http://www.apa.org/releases/ interadd.html

Griffiths, M. D. (1995a, April 7). Netties anonymous. Times Higher Educational Supplement, p. 18.

Griffiths, M. D. (1995b). Technological addictions. Clinical Psychology Forum, 76, 14-19.

GRIFFITHS, M. D. (1996). Internet "addiction": An issue for clinical psychology? Clinical Psychology Forum, 97, 32-36.

GRIFFITHS, M. D. (1997). Psychology of computer use: XLIII. Some comments on 'Addictive use of the Internet' by Young. Psychological Reports, 80, 81-82.

GrIFFITHS, M. D. (1998). Internet addiction: Does it really exist? In J. E. Gackenbach (Ed.), Psychology and the Internet: Intrapersonal, interpersonal, and transpersonal implications (pp. 61-75). New York: Academic Press. 
HEALY, J. M. (1998). Failure to connect: How computers affect our children's minds, for better and worse. New York: Simon \& Schuster. HENDERSON, D. (1996, March 1). I'm Kris: I'm a Webaholic: Use of online computer services can be addictive. Seattle Times, pp. A1, A9.

JACOBSON, H. B., \& RoucEK, J. S. (1959). Automation and society. New York: Philosophical Library.

KATZ, J. E., \& ASPDEN, P. (1997). A nation of strangers? Communications of the $A C M, 40,81-86$.

KinG, J. L., \& KRAEMER, K. L. (1995). Information infrastructure, national policy, and global competitiveness. Information Infrastructure \& Policy, 4, 5-28.

Kraut, R., Patterson, M., Lundmark, V., Kiesler, S., MukopadHYAY, T., \& SCHERLIS, W. (1998). Internet paradox: A social technology that reduces social involvement and psychological wellbeing? American Psychologist, 53, 1017-1031.

O'NeILL, M. (1995, March 8). The lure and addiction of life on line. New York Times, pp. B1, B7.

ORZACK, M. H. (1998). Computer addiction services [On-line]. Available: http://www.cse.stanford.edu/classes/cs201/current/Projects/ computer-addiction/index.html

RHEINGOLD, H. (1993). The virtual community: Homesteading on the electronic frontier. Reading, MA: Addison-Wesley.

ROBERTS, L. (1997). Electronic opium: Computer addiction in the Internet age [On-line]. Available: http://www.cse.stanford edu/classes/ cs201/current/Projects/computer-addiction/index.html

ShotTon, M. A. (1989). Computer addiction? London: Taylor \& Francis.
SHOtтon, M. A. (1991). The costs and benefits of computer addiction. Behaviour \& Information Technology, 10, 219-230.

SLEEK, S. (1998). Isolation increases with Internet use. American Psychological Association Monitor, 29, 1, 30.

Sproull, L., \& FaraJ, S. (1995), Atheism, sex, and databases: The Net as a social technology. In B. Kahin \& J. Keller (Eds.), Public access to the Internet (pp. 62-81). Cambridge, MA: MIT Press.

Young, K. S. (1996). Psychology of computer use: XL. Addictive use of the Internet: A case that breaks the stereotype. Psychological Reports, 79, 899-902.

\section{NOTE}

1. We elected to include three items in the survey that focused on personality traits out of convenience, rather than administering a lengthy and separate personality inventory. On the basis of this small amount of evidence, we do not make the causal argument that shyness/introversion causes computer/Internet addiction or the component related to their use for sexual gratification. The data merely show that a preponderance of the surveys produced a correlation with these two items on Factor 3. Moreover, the rationale for including these items in the present survey was based on their having been identified in the literature as making a functional contribution to the symptomology. Having identified this correlation, future research with individuals whose behavioral profile is congruent with the variables loading onto Factors 1 and 3 should use a valid personality inventory to scrutinize these individual differences.

\section{APPENDIX}

Computer Use Survey

I am: $\diamond$ male $\diamond$ female

Age :

Highest schooling completed: (check only the highest)

$\checkmark$ elementary school

$\checkmark$ high school

$\checkmark$ some college

$\checkmark$ college graduate

$\checkmark$ master's degree

$\checkmark$ doctorate or equivalent

I am primarily (full time): (check only one)

$\diamond$ a student

$\checkmark$ employed part-time nonprofessional

$\checkmark$ employed full-time nonprofessional

$\checkmark$ employed part-time professional

$\checkmark$ employed full-time professional

$\checkmark$ self-employed

$\diamond$ unemployed

I belong to

$00-2$

(number of) clubs or organizations on or off the Internet.

I connect to the Internet through my

$\checkmark$ school or university

$\diamond$ work

$\diamond$ public server or freenet

$\diamond$ national Internet service provider (America On Line, CompuServe, etc.)

$\checkmark$ local commercial Internet service provider

I spend an average of hours per week on the Internet, e.g., WWW, email, etc.

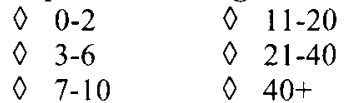


APPENDIX (Continued)
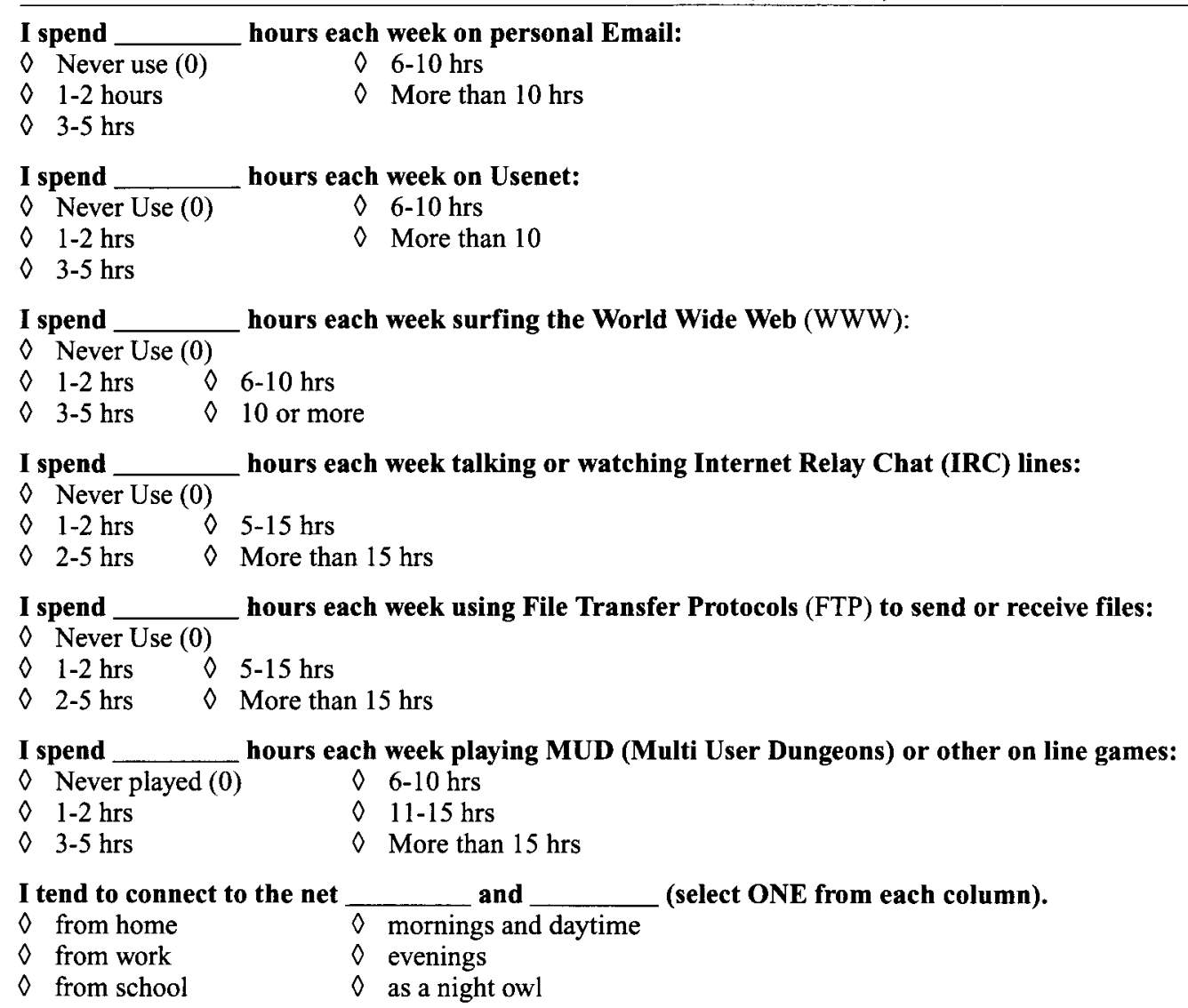

The main reason(s) I use the Internet is(are) (check all that apply):

$\checkmark$ school related assignments

$\checkmark$ for my own personal growth and development

$\checkmark$ for software

$\checkmark$ to talk to friends far away

$\checkmark$ to meet new people

$\checkmark$ to talk to others who share my interests

$\checkmark$ to keep track of new developments in areas of personal interest (hobby, culture, news, weather)

$\checkmark$ to make purchases

$\checkmark$ cybersex

0 other (specify)

I consider myself

$\checkmark$ not at all a

$\diamond$ a moderate

$\diamond$ an expert

\section{My relationship status is best described as}

$\diamond$ never married

$\checkmark$ not married but in a significant, long-term relationship

$\checkmark$ married

$\diamond$ separated

$\checkmark$ divorced

$\checkmark$ not interested in a relationship at this time

\section{I have been using the Internet for:}

$\diamond$ 0-3 months

$\checkmark$ 4-6 months

$\checkmark$ 7-12 months

$\diamond 1-2$ years

$\checkmark$ 2-5 years

0 more than 5 years 


\section{APPENDIX (Continued)}

The amount of time I spend on computer(s) has caused me to

$\diamond$ gain weight

$\diamond$ lose weight

$\diamond$ no change

\section{Circle (T)rue or (F)alse to each of the following computer/Internet-related experiences.}

1. My first computer experience was with video games.

2. I have spent more than three continuous hours on-line at least twice.

3. More than once, I have been late for appointments because I was on-line.

4. I know most of my friends from the Internet.

5. I would rather spend an evening on-line than going out with friends.

6. More than once, I have gotten less than 4 hours of sleep in a night because I was on-line (excluding completing homework, studying, etc.).

7. More than once, I have missed a meal because I was on-line so long.

8. I have made arrangements to rendezvous with someone I knew only from the Internet.

9. I have used the Internet to help me feel better when I was sad, down or depressed.

10. My grades have dropped since I have been on-line.

11. There have been times when I have dialed a telephone number or attempted to connect to a remote site, only to find that the line was busy or the site was down.

12. I have used the Internet to talk to others at times when I was feeling lonely.

13. I find it easier to talk to people on the Internet than in person.

14. I have attempted to resolve an interpersonal conflict electronically instead of face-to-face.

15. I have shared a deep, dark secret with a person I met on the Internet.

16. I have been misled for at least one month as to someone's gender on the Internet.

17. I have more fun with people I know on the Internet than those I know in person.

18. When I wake up, the first thing I want to do is get on the computer.

19. I have never watched television.

20. I spend so much time on the computer, that 1 lose track of time and forget to eat.

21. People say I am shy.

22. Social relationships are important to me.

23. I have voluntarily gone more than 3 days without connecting in the past 3 months.

24. I have been told that I spend too much time on my computer or on-line.

25. I have been accused of being a computer "nerd" or computer "junkie".

26. I have used Internet resources intended for adults only.

27. I have rearranged my social plans to give myself time to be on-line.

28. I have always been more introverted than extroverted.

29. If it weren't for my computer, I would not have any fun at all.

30. I laugh, smile and feel the happiest when I am on the computer.

31. I have tried to hide from others the amount of time I am actually on-line.

32. I never experience eye strain when using the computer.

33. I have never met anyone who wears glasses or contacts.

34. I have gotten a bill for on-line services that was at least twice what I expected.

35. I have gone into debt for connection fees.

36. I have gotten into trouble with my employer because of the Internet.

37. I have missed at least 3 classes that $I$ intended to attend because I was on-line.

38. At times, I have noticed changes in my eating habits because of the Internet.

39. I have never had an argument with my significant-other because of my Internet use.

40. More than 5 days have passed since I l ast showered.

41. I have unsuccessfully attempted to spend less time on-line.

42. My exercise habits have changed since I found the net.

43. Net friends have introduced me to things I would never have done before.

44. More than once, I have gotten physically aroused while I was on-line.

45. People say my personality has changed since I went on-line.

46. I have gotten into trouble with my employer/school for Internet related activities.

47. Only my Internet friends really know who I really am.

48. I prefer to have an intimate on-line relationship with someone I met on the Internet than someone in person.

49. I have found information or software that I needed free on the Internet.

50. I have borrowed money to cover my connection fees.

51. I find the Internet to be impersonal.

52. My last girlfriend/boyfriend complained about my on-line usage.

53. I have had to buy a better computer since I found the Internet.

54. I feel I have an extroverted personality.

55. I routinely cut my sleep hours short to spend more time on-line.

T F

T F

T $\mathbf{F}$

T F

T F

T $\mathrm{F}$

T F

T F

T F

T F

T $\quad \mathrm{F}$

T F

T F

T F

T F

T F

T $\mathrm{F}$

T F

T F

T F

T $\mathrm{F}$

T F

T F

T F

T F

T F

T F

T F

T F

T F

T F

T F

T F

T F

T F

T F

T $\mathrm{F}$

T F

T F

T F

T F

T F

T F

T F

T F

T F

T $\mathrm{F}$

T $\mathrm{F}$

T F

T F

T F

T $\mathbf{F}$

T F

T F

T F 


\section{APPENDIX (Continued)}

56. I occasionally have dreams of being on-line.

57. If it has been a while since I last logged on, I find it hard to stop thinking about what will be waiting for me when I do.

58. I am concerned that government attempts to control the Internet will impact negatively on the things I like to do on the net.

59. I find it easier to talk face-to-face to a person than to chat on the Internet.

60. My work and/or performance has not deteriorated since I started using the Internet.

61. I have no desire to meet my Internet friends in person.

62. I would not feel irritated if I could not use my computer today.

63. If computer access was shut down in my city for 48 hours, I would drive to the nearest city for access.

64. I have downloaded or viewed sexually oriented pictures on the Internet.

65. I have used the net for gambling purposes.

66. I often leave my computer connected to the Internet so I won't have trouble getting on again later.

67. I write computer programs beyond what is required for my job or my schoolwork.

68. I have attempted to hack my way into a system at least once.

69. After spending hours at a computer, I have sometimes experienced some disorientation, headaches, or visual effects.

70. I like to discover new Internet features or activities and be among the first to use them.

71. I have ordered merchandise over the Internet.

72. I ask questions on the Internet that I could easily find the answers to in the library.

73. I have sent or received coded or encrypted messages over the Internet at least once.

74. Knowing something that no one else knows is something I enjoy a lot. 